\title{
Decreased serum fibroblast growth factor 19 level is a risk factor for type 1 diabetes
}

\author{
Jingyi Hu${ }^{1,2,3 \#}$, Yingxin Tang ${ }^{1,2,3 \#}$, Hui Liu ${ }^{1,2,3}$, Yanhua $\mathrm{Li}^{1,2,3}, \mathrm{Xia}_{\mathrm{Li}^{1,2,3}}$, Gan Huang ${ }^{1,2,3}$, Yang Xiao ${ }^{1,2,3}$, \\ Zhiguang Zhou ${ }^{1,2,3}$
}

${ }^{1}$ Department of Metabolism and Endocrinology, The Second Xiangya Hospital, Central South University, Changsha, China; ${ }^{2}$ National Clinical Research Center for Metabolic Diseases, Changsha, China; ${ }^{3}$ Key Laboratory of Diabetes Immunology, Central South University, Ministry of Education, Changsha, China

Contributions: (I) Conception and design: J Hu, Y Tang, Y Xiao, Z Zhou; (II) Administrative support: X Li, G Huang, Y Xiao, Z Zhou; (III) Provision of study materials or patients: H Liu, X Li, Y Xiao, Z Zhou; (IV) Collection and assembly of data: H Liu, G Huang, X Li, G Huang; (V) Data analysis and interpretation: J Hu, Y Tang, Y Xiao; (VI) Manuscript writing: All authors; (VII) Final approval of manuscript: All authors.

\#These authors contributed equally to this work.

Correspondence to: Yang Xiao; Zhiguang Zhou. Department of Metabolism and Endocrinology, The Second Xiangya Hospital, Central South University, Changsha, China. Email: xiaoyang29@csu.edu.cn; zhouzhiguang@csu.edu.cn.

Background: Increasing evidence suggests that fibroblast growth factor 19 (FGF19) is a regulator of glucose metabolism and may provide a new therapeutic target for type 1 diabetes (T1D). However, the clinical relevance of FGF19 in T1D remains unclear. In this study, we examined the relationship between the serum FGF19 concentration and T1D.

Methods: This study included 81 newly diagnosed T1D patients and 80 sex- and age-matched healthy controls. The correlation between the FGF19 concentration and clinical characteristics of T1D patients and healthy controls was investigated. Logistic regression analysis was performed to determine whether levels of FGF19 were independently associated with T1D.

Results: The fasting serum FGF19 levels in the T1D group were significantly lower than those in the control group [159.9 (100.0-272.7) vs. 205.0 (126.9-307.9) pg/mL, P=0.008]. In all subjects, serum FGF19 levels were negatively correlated with fasting blood glucose $(\mathrm{FBG})(\mathrm{r}=-0.192, \mathrm{P}=0.015)$. In the control group, serum FGF19 levels were positively correlated with total cholesterol (TC) ( $\mathrm{r}=0.338, \mathrm{P}=0.002)$ and lowdensity lipoprotein cholesterol (LDL-c) $(\mathrm{r}=0.300, \mathrm{P}=0.007)$. In addition to sex and body mass index (BMI), FGF19 was an independent impact factor for T1D [odds ratio (OR) =0.541, P=0.023; adjusted for sex, age, BMI, presence of hypertension, and presence of dyslipidemia].

Conclusions: Low serum FGF19 level is associated with T1D, which could serve as a risk factor for T1D.

Keywords: Type 1 diabetes (T1D); fibroblast growth factor 19 (FGF19); risk factor

Submitted Jul 12, 2020. Accepted for publication Dec 16, 2020.

doi: 10.21037/atm-20-5203

View this article at: http://dx.doi.org/10.21037/atm-20-5203

\section{Introduction}

Type 1 diabetes (T1D), a chronic disorder caused by autoimmune destruction of islet $\beta$ cells $(1,2)$, usually begins early in life; moreover, T1D is associated with serious shortterm and long-term consequences that place a heavy burden on patients (3). The global incidence of T1D has been increasing yearly (4). However, the pathogenesis of T1D remains unclear and the current therapy for T1D is limited.

Fibroblast growth factor 19 (FGF19), belongs to the endocrine FGF19 subfamily and differs from canonical FGF molecules, which function as circulating hormones. Previous studies have shown that FGF19 plays an indispensable role in bile acid (BA), glucose, and lipid metabolism (5) and could be a potential molecule for treating T1D. In 
this regard, scientists have found that FGF19 can activate the Ras and extracellular signal-regulated protein kinase (ERK) signaling pathways to stimulate hepatic glycogen production in streptozotocin (STZ)-induced diabetic mice $(5,6)$, and that intraventricular injection of FGF19 in a rat model of T1D can reduce hepatic glucose production (HGP), improve blood glucose and reduce insulin resistance $(7,8)$. In humans, recent publications have reported that FGF19 may be a major protector of islet $\beta$ cell function (9). However, the relationship between FGF19 and T1D has not yet been elucidated.

In this study, we measured circulating levels of FGF19 in T1D patients and age- and sex-matched healthy control subjects, aiming to investigate whether serum FGF19 levels change in T1D patients compared to those in healthy controls and to provide a possible explanation for the role of FGF19 during T1D development. We present the following article in accordance with the Materials Design Analysis Reporting (MDAR) checklist (available at http:// dx.doi.org/10.21037/atm-20-5203).

\section{Methods}

\section{Participants}

We conducted a cross-sectional study of the Chinese Han population. Eighty-one newly diagnosed T1D patients were recruited from the Second Xiangya Hospital of Central South University between September 1999 and November 2010 (Figure 1). The inclusion criteria for patients in the T1D group were as follows: (I) clinical characteristics meeting the 1999 World Health Organization (WHO) criteria (10); (II) insulin dependence within 6 months after diagnosis; (III) the presence of at least one positive autoantibody (GADA, IA-2A, ZnT8A); (IV) the presence of diabetic ketosis or ketoacidosis at the onset of the disease; and $(\mathrm{V})$ duration of diabetes $<1$ year (11). The exclusion criteria were as follows: severe chronic cardiovascular, cerebrovascular, renal or liver disease; abnormalities in liver function, renal function or routine blood tests; hyperthyroidism; other autoimmune diseases; or malignant tumors. Age- and sex-matched controls were recruited from children in the community who came for health screening at the Children's Health Center of the Second Xiangya Hospital, Central South University (12). Healthy controls were also collected at the same time (Figure 1). Subjects with diabetes or a family history of diabetes, thyroid disease, other autoimmune diseases, or any other acute or chronic diseases were excluded from the group of healthy controls. The diagnosis of dyslipidemia was based on the 2001 American National Cholesterol Education Program Adult Treatment Panel III (NECP-ATP III) standard (13). Hypertension was diagnosed when systolic blood pressure (SBP) was $\geq 140$ or diastolic blood pressure (DBP) was $\geq 90 \mathrm{mmHg}$ or if patients were taking antihypertensive medications (14).

\section{Research design, anthropometric and biochemical measurements}

Age, sex, and medical history of all subjects were recorded, and height, weight, waist circumference (WC), hip circumference (HC), and blood pressure were measured according to standard measurement methods (15). After 8 hours of fasting, venous blood was collected in the morning from all participants. Serum samples separated from the collected venous blood were used to measure biochemical parameters, and the remaining serum was stored at -80 degrees Celsius. Fasting blood glucose (FBG), total cholesterol (TC), triglyceride (TG), high-density lipoprotein cholesterol (HDL-c) and low-density lipoprotein cholesterol (LDL-c) were measured by a Hitachi 7170 analyzer (Boehringer Mannheim, Mannheim, Germany) (16). Fasting C-peptide (FCP) was measured by an automated chemical analyzer (Bayer Centaur Immunoassay System, Germany). An enzyme-linked immunosorbent assay (ELISA) kit was used to measure serum FGF19 levels according to the manufacturer's instructions (BioVendor, Cat. No.: RD191107200R). The minimum detection limit was $4.8 \mathrm{pg} / \mathrm{mL}$, and the intra- and inter-assay coefficients of variation were $6.0 \%$ and $7.5 \%$, respectively.

\section{Sample size}

In this study, unpaired Student's $t$-tests were used to calculate the sample size. Previous studies estimated that the standard deviation (SD) of fasting serum FGF19 levels of healthy controls was approximately 80 , and it was estimated that a difference of 40 in the FGF19 level between the two groups was significant $(17,18)$. For a power of 0.8 and $\alpha$ of 0.05 , the number of participants required per group was 64 after formula calculation (19).

$$
n=2\left[\frac{\left(u_{\alpha}+u_{\beta}\right)}{\delta / \sigma}\right]^{2}+\frac{1}{4} u_{\alpha}^{2}
$$


A

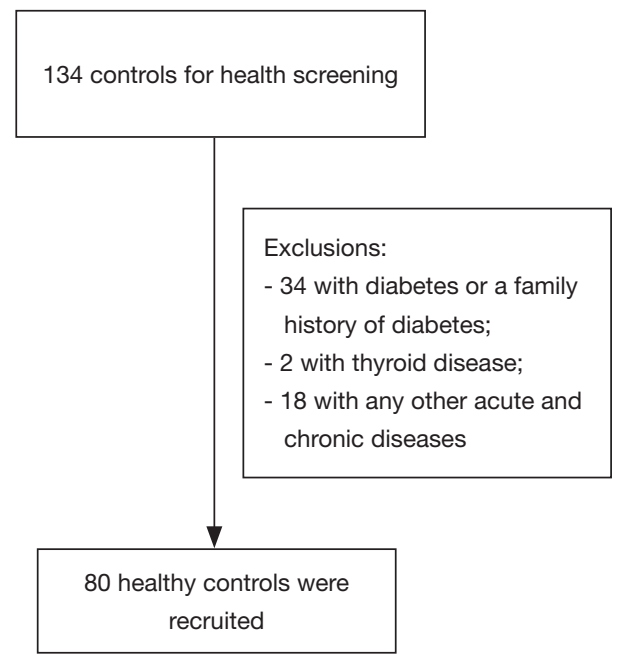

B

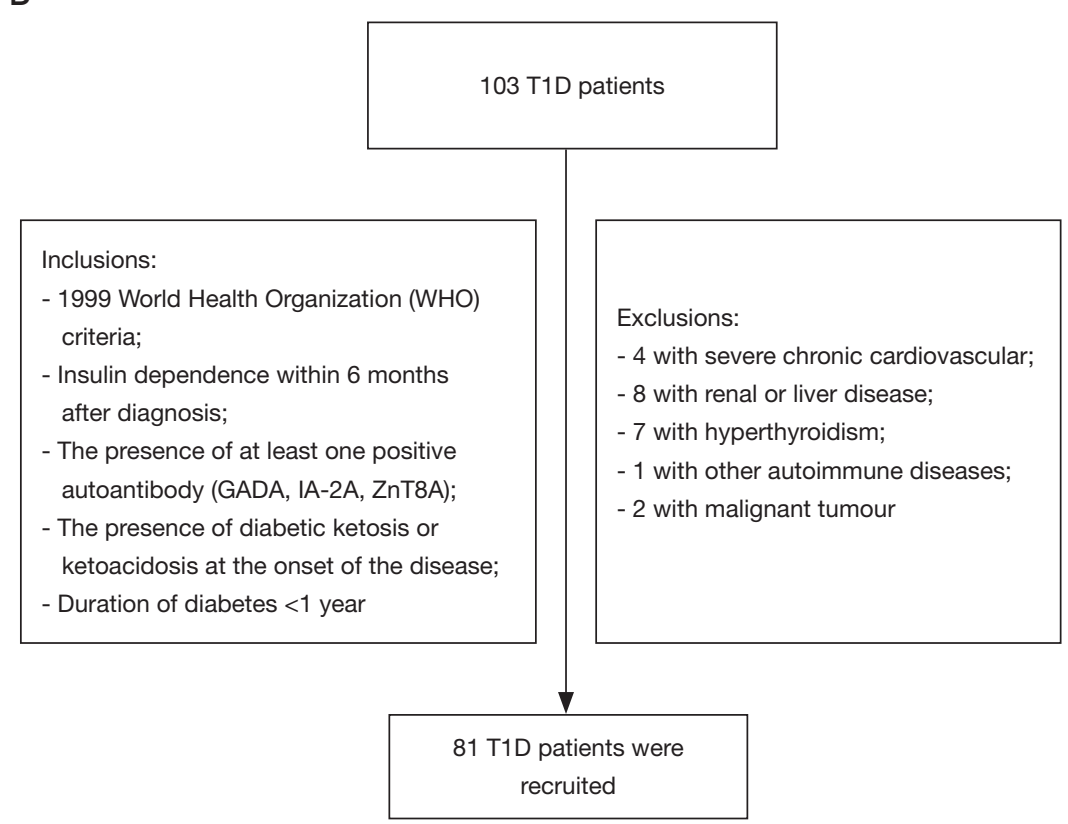

Figure 1 Flow charts of T1D patients and control subjects recruited to the study. T1D, type 1 diabetes.

\section{Statistical analyses}

Statistical analyses of all data were performed with SPSS 25.0 statistical software. Normally distributed data, as determined by the Kolmogorov-Smirnov test, were presented as mean $\pm \mathrm{SD}$, and non-normally distributed data were presented as median and interquartile range (IQR). Unpaired Student's $t$-tests and nonparametric tests were used to compare differences in normally distributed and non-normally distributed data between two groups, respectively. The chi-square test was used to compare differences in categorical variables between groups. Spearman correlation analysis was performed to analyze the association between serum FGF19 levels and other variables. Logistic regression analysis was performed to investigate independent impact factors of T1D. $\mathrm{P}$ values $<0.05$ were considered to be statistically significant.

\section{Ethical statement}

All participants were recruited, and all samples were collected after appropriate informed consent was provided by all participants or their guardians. The study was approved by the ethics committee of the Second Xiangya Hospital of Central South University (No.: 2019-Research-40) and was conducted in accordance with the Declaration of Helsinki (as revised in 2013).

\section{Results}

The clinical characteristics of the 161 subjects are shown in Table 1. The age and sex of the T1D group were comparable to those of the controls $(\mathrm{P}>0.05)$. Compared with control subjects, patients with T1D had higher FBG, TC, TG, LDL-c, and creatinine (Cr) levels but lower body mass index (BMI), waist-hip ratio (WHR), SBP, and FCP levels. In addition, serum FGF19 levels of T1D patients were significantly lower than those of healthy controls [159.9 (100.0-272.7) vs. 205.0 (126.9-307.9) pg/mL, $\mathrm{P}=0.008$; Figure 2].

In all subjects, serum FGF19 levels were negatively correlated with FBG and LDL-c $(\mathrm{r}=-0.192, \mathrm{P}=0.015$; $\mathrm{r}=0.165, \mathrm{P}=0.037)$. There was no significant correlation between serum FGF19 levels and age, BMI, WHR, FBG, FCP, TG, HDL-c, SBP, diastolic DBP, blood urea nitrogen (BUN) or Cr, in either the control group or the T1D group $(\mathrm{P}>0.05)$. In addition, no correlation was found between FGF19 levels and the duration of T1D $(\mathrm{P}>0.05)$. Although no significant differences were observed between FGF19 levels and lipid profiles in the T1D group, FGF19 levels were positively correlated with TC and LDL-c $(r=0.338$, 
Table 1 Clinical characteristics among control subjects and T1D subjects

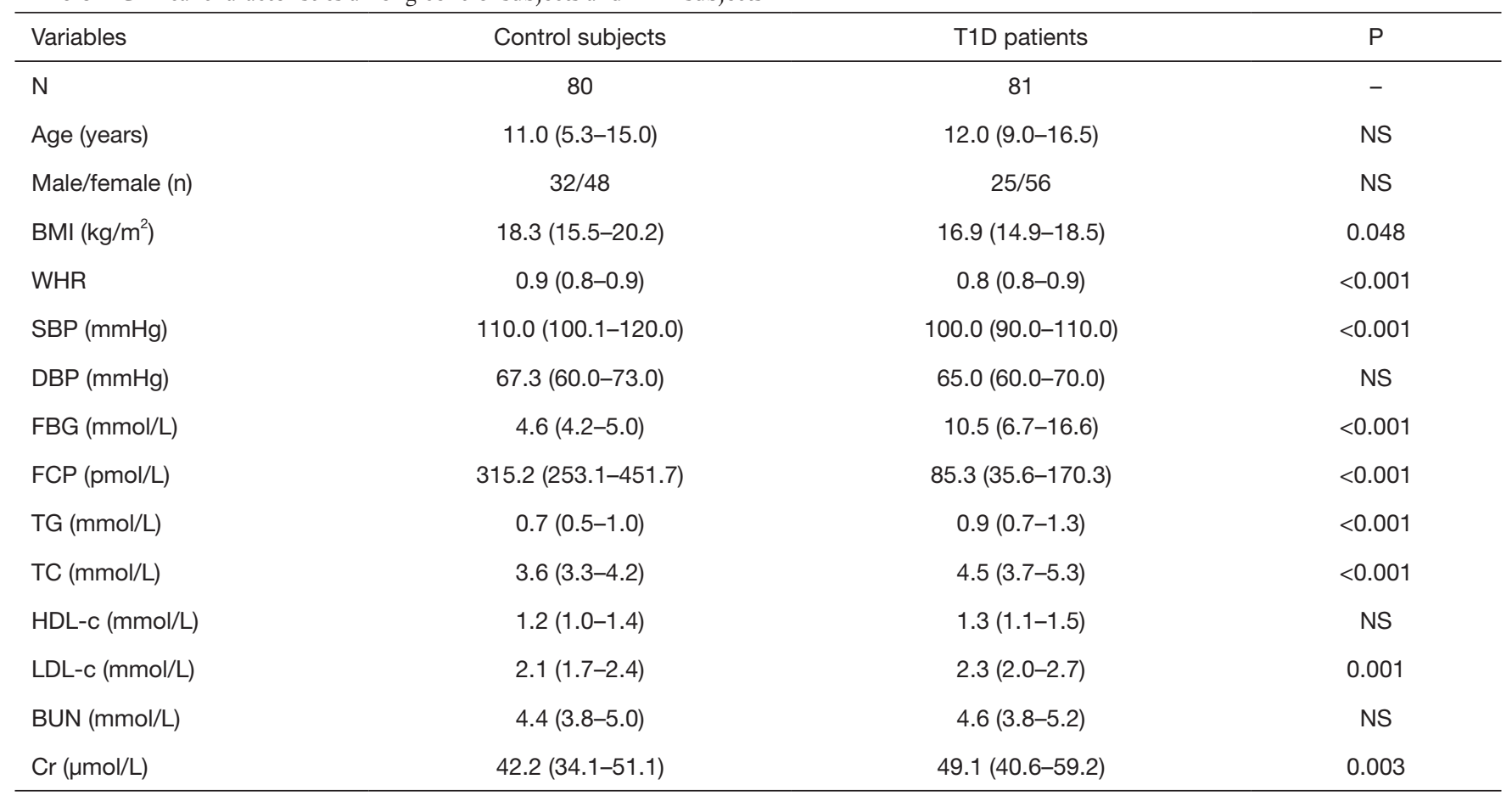

Data are presented as mean \pm SD or median (interquartile range). BMI, body mass index; WHR, waist-hip ratio; SBP, systolic blood pressure; DBP, diastolic blood pressure; FBG, fasting blood glucose; FCP, fasting C-peptide; TC, total cholesterol; TG, triglycerides; HDL-c, high-density lipoprotein cholesterol; LDL-c, low-density lipoprotein cholesterol; BUN, blood urea nitrogen; Cr, creatinine.

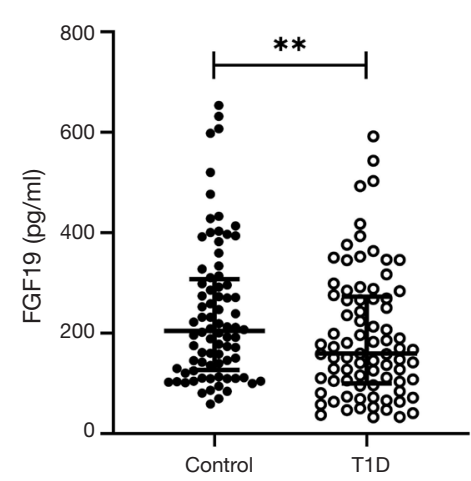

Figure 2 Comparison of serum FGF19 levels in the control group and T1D group. FGF19, fibroblast growth factor 19; T1D, type 1 diabetes. ${ }^{* *}, \mathrm{P}<0.01$.

$\mathrm{P}=0.002 ; \mathrm{r}=0.300, \mathrm{P}=0.007$; Figure 3 ) in the control group. After adjustment for sex and age, FGF19 levels remained positively correlated with $\mathrm{TC}(\mathrm{r}=0.266, \mathrm{P}=0.018$; Table 2$)$.

To assess the relationship between the serum concentrations of FGF19 and the development of T1D, unadjusted and multivariate adjusted logistic regression analyses were performed. Unadjusted logistic regression analysis demonstrated that the FGF19 level was a significant factor associated with the presence of T1D [odds ratio $(\mathrm{OR})$ $=0.511, \mathrm{P}=0.009$; Table 3). As shown in Table 3, using T1D as the dependent variable, FGF19 $(\mathrm{OR}=0.541, \mathrm{P}=0.023$; Table 3), together with age $(\mathrm{OR}=1.094, \mathrm{P}=0.017)$, and $\mathrm{BMI}$ $(\mathrm{OR}=0.871, \mathrm{P}=0.019)$, were independently associated with the presence of T1D.

\section{Discussion}

In our study, we found that T1D patients had lower serum FGF19 levels than healthy controls, and low serum FGF19 levels were associated with the occurrence of T1D. To the best of our knowledge, this is the first study to focus on the association between FGF19 levels and T1D.

T1D is characterized by destruction of islet $\beta$ cells, which leads to insulin deficiency and glucose metabolic disorders $(20,21)$. The discovery of metabolic mechanisms related to T1D can help to better understand its pathogenesis and improve the treatment of T1D. FGF19 plays an indispensable role in regulating glucose metabolism $(22,23)$. 
Table 2 Spearman correlations of serum FGF19 levels and metabolic parameters

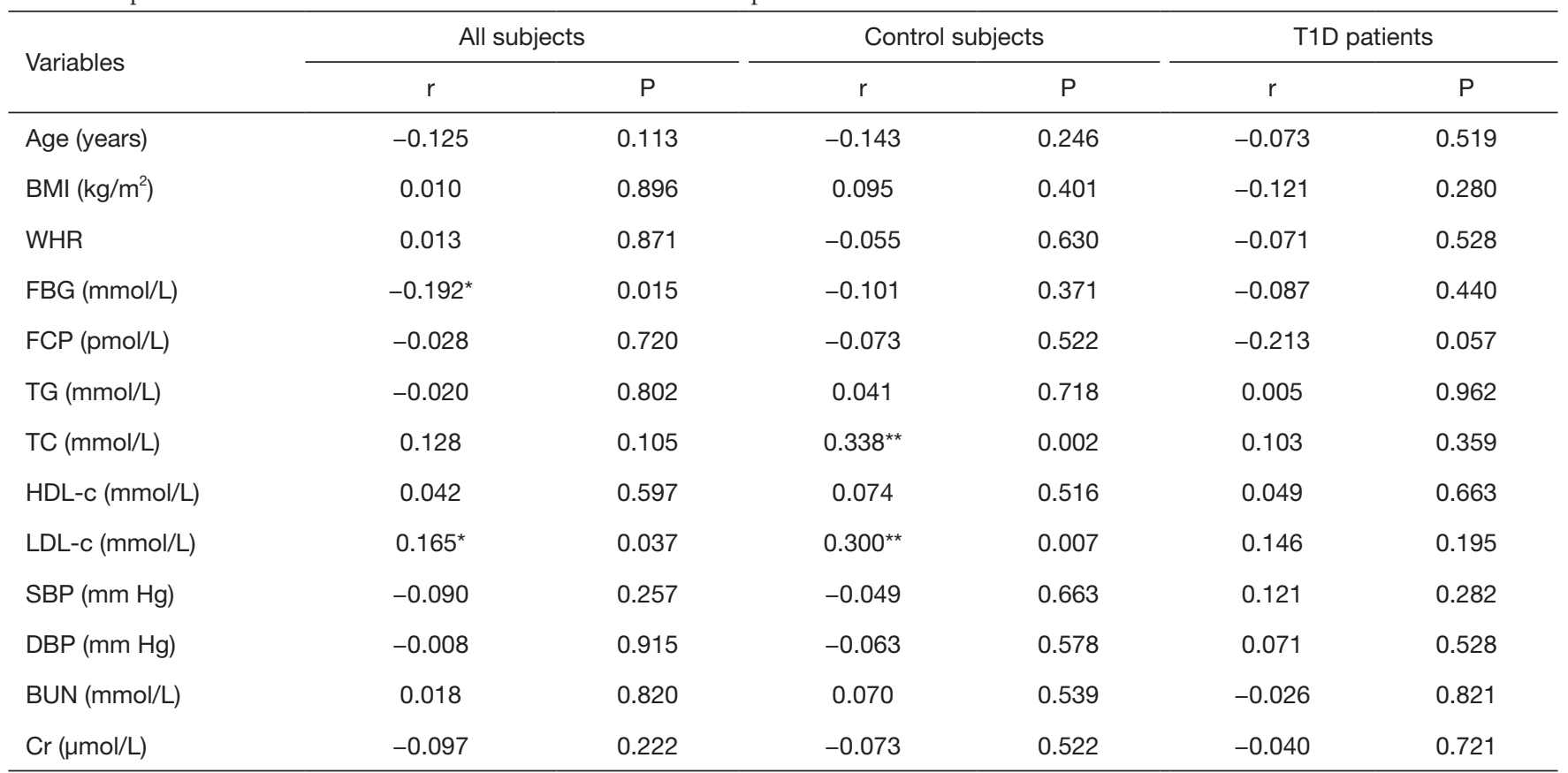

BMI, body mass index; WHR, waist-hip ratio; SBP, systolic blood pressure; DBP, diastolic blood pressure; FBG, fasting blood glucose; FCP, fasting C-peptide; TC, total cholesterol; TG, triglycerides; HDL-c, high-density lipoprotein cholesterol; LDL-c, low-density lipoprotein cholesterol; BUN, blood urea nitrogen; Cr, creatinine.

A

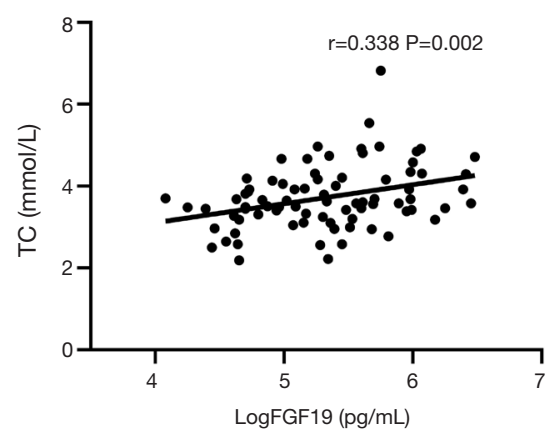

B

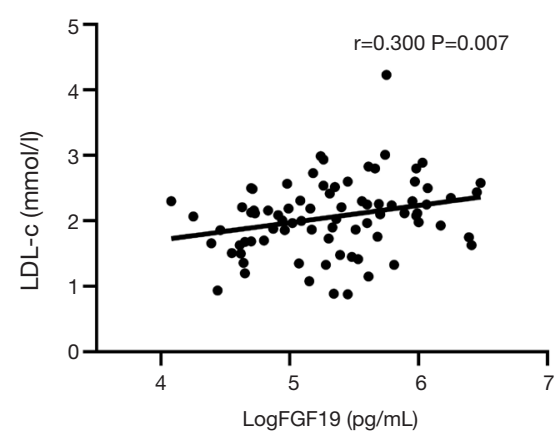

Figure 3 Serum FGF19 levels were positively correlated with TC and LDL-c. (A) Correlation between FGF19 and TC in healthy controls, $\mathrm{TC}=1.25+0.46^{*} \ln F G F 19$. (B) Correlation between FGF19 and LDL-c in healthy controls, LDL-c $=0.65+0.26^{*} \ln F G F 19$. FGF19, fibroblast growth factor 19; TC, total cholesterol; LDL-c, low-density lipoprotein cholesterol.

The pancreas is the target organ of FGF19 $(9,24)$. However, the association between FGF19 and T1D remains unclear.

In this study, we found that patients with T1D had lower serum FGF19 levels than healthy controls. In STZinduced diabetic mice, FGF19 can stimulate glycogen synthase activity through mitogen-activated protein kinase
(MAPK) signaling pathways to increase hepatic glycogen synthesis (5). In addition, FGF19 can inhibit the activity of cAMP regulatory element-binding protein (CREB) to downregulate glucose synthesis in mice $(23,25)$. Perry et al. found that FGF19 can reduce HGP by inhibiting the hypothalamic-pituitary-adrenal axis in a rat model, 
Table 3 Logistic regression analysis of risk factors for T1D

\begin{tabular}{|c|c|c|c|c|}
\hline Variables & \multicolumn{2}{|c|}{ Simple } & \multicolumn{2}{|c|}{ Multiple } \\
\hline FGF19 ${ }^{\dagger}$ & $0.511(0.309-0.847)$ & 0.009 & $0.541(0.319-0.918)$ & 0.023 \\
\hline Sex & 1.493 (0.780-2.860) & 0.227 & 1.094 (1.016-1.179) & 0.017 \\
\hline Age & 1.663 (0.978-2.829) & 0.061 & $1.730(0.833-3.591)$ & 0.141 \\
\hline Presence of hypertension & $2.026(0.360-11.386)$ & 0.423 & 3.593 (0.490-26.338) & 0.208 \\
\hline Presence of dyslipidemia & 1.077 (0.580-1.998) & 0.814 & $0.807(0.399-1.630)$ & 0.549 \\
\hline
\end{tabular}

${ }^{\dagger}$, log transformed before analysis. Variables included in the adjusted model were sex, age, BMI, presence of hypertension, presence of dyslipidemia and FGF19. FGF19, fibroblast growth factor 19. BMI, body mass index.

indicating that FGF19 has a noninsulin hypoglycemic effect in T1D (7). Other studies have shown that the improvement of glucose metabolism by FGF19 involves the inhibition of AGRP/NPY neuronal activity (26). In humans, Hwang et al. found that patients with T1D had lower levels of hepatic glycogen than healthy controls (27). In this regard, we may conclude that a reduction in FGF19 levels in T1D patients could have resulted in low hepatic glycogen synthesis, which is related to imbalanced glycemic control.

Our research suggests that serum FGF19 levels are associated with the presence of T1D, and this association may be related to the protective effect of FGF19 on islet $\beta$ cells. Dysfunction of islet $\beta$ cells is closely related to oxidative stress, and FGF19 can alleviate stressed $\beta$ cells and preserve their function $(9,28)$. FGF19 has been shown to induce antioxidant responses in STZ-induced diabetic mice via the AMP-activated protein kinase (AMPK)/nuclear erythroid factor 2-related factor 2 (NRF2)/heme oxygenase-1 (HO-1) signaling pathway (29). Another possibility is that FGF19 may regulate islet $\beta$ cell secretion and insulin levels through the BA-farnesoid X receptor (FXR)-FGF19 axis and glucagon-like peptide-1 (GLP-1) secretion (9). In addition, the intestinal microbiota matures early in life and affects the immune response to mediate the development of T1D (30,31). The intestinal microbiota also regulates BA homeostasis by stimulating the expression of FGF19 through intestinal nuclear FXR (32-34). BAs have strong antibacterial activity that can directly regulate the intestinal microbiota (32). Furthermore, FGF19 serves as a link between the intestine and glucose metabolism. More research is needed to clarify the specific mechanism of the role of FGF19 in the metabolic balance of "intestinal microbiota-BA". The aforementioned evidence may lead to the speculation that
FGF19 could be a new therapeutic target for T1D.

Moreover, increasing evidence in humans has revealed a significant correlation between FGF19 and glucose metabolism disorders. Three previous studies found that serum FGF19 levels in patients with type 2 diabetes and patients with impaired FBG were lower than those in controls with normal glucose tolerance $(9,35,36)$. Consistent with previous studies, the serum FGF19 levels of all participants were negatively correlated with FBG in our study $(35,37)$. However, no correlation was found between patients with T1D and healthy controls. We speculate that this may be due to the small sample size.

Previous studies also found that FGF19 was positively correlated with HDL-c and negatively correlated with TG in patients with metabolic syndrome (38) and that FGF19 was negatively correlated with LDL-c in healthy controls (39). An FGF19 analog (NGM282) was used in clinical trials on healthy individuals, which resulted in increased serum cholesterol, HDL-c, and LDL-c levels and reduced TG levels (40). In our study, T1D patients had higher TC, TG, and LDL-c levels than healthy controls, and in the control group, FGF19 was positively correlated with TC and LDL-c. After adjusting for age and sex, FGF19 was positively correlated only with TC. One possible explanation is that FGF19 can affect cholesterol metabolism by inhibiting the rate-limiting enzyme in the synthesis of bile, cholesterol $7 \alpha$-hydroxylase (CYP7A1) $(23,41)$. One-third of the catabolism of cholesterol in the human body is achieved by conversion to BAs $(42,43)$. Phosphorylation of liver FXR by the nonreceptor tyrosine kinase Src, induced by FGF19, maintained cholesterol homeostasis (44). In addition, FGF19 can increase fatty acid $\beta$ oxidation by inhibiting acetyl-CoA carboxylase 2 
(ACC2) (23). These factors can also affect the relationship between FGF19 and TC, HDL-c, LDL-c, and TG.

Serum FGF19 levels were not associated with age or sex in either the control group or the T1D group, which was consistent with previous studies $(37,45)$. Previous studies found that serum FGF19 levels are lower in obese patients than in lean people and healthy controls (46). Friedrich et al. found that BMI was negatively associated with the fasting FGF19 level (47). In this study, we did not find a significant correlation between FGF19 and BMI or WHR. A possible explanation is that the subjects we recruited (healthy controls and T1D patients) were thinner than the average population and did not represent the general population. Therefore, we could not determine the correlation between FGF19 and BMI in this study.

This study has several limitations. First, only changes in fasting serum FGF19 levels were investigated. While digestion has a significant effect on serum FGF19 levels (48), it also has effects on blood glucose, insulin, and C-peptide levels. The relationship between postprandial FGF19 levels and these metabolic indicators is unclear and needs further exploration. Second, this is a cross-sectional study with a limited sample size, so it does not explain the causal relationship between decreased serum FGF19 levels and T1D. The specific role of decreased serum FGF19 levels in the pathogenesis of T1D requires further research to validate the conclusions of this study and clarify other issues.

\section{Conclusions}

Serum FGF19 levels in patients with T1D were lower than those in healthy controls. Serum FGF19 is associated with T1D, indicating that decreased FGF19 is a risk factor for T1D.

\section{Acknowledgments}

This study was edited by American Journal Experts (AJE) Language Editing Service.

Funding: This work was supported by the National Key Research and Development Program of China [2018YFE0114500 to YX], the Natural Science Foundation of Hunan Province for Excellent Young Scholars [2020JJ3056 to $\mathrm{YX}$, the National Natural Science Foundation of China [81670772 to YX, 81820108007 to ZZ].

\section{Footnote}

Reporting Checklist: The authors have completed the MDAR reporting checklist. Available at http://dx.doi.org/10.21037/ atm-20-5203

Data Sharing Statement: Available at http://dx.doi. org/10.21037/atm-20-5203

Peer Review File: Available at http://dx.doi.org/10.21037/ atm-20-5203

Conflicts of Interest: All authors have completed the ICMJE uniform disclosure form (available at http://dx.doi. org/10.21037/atm-20-5203). The authors have no conflicts of interest to declare.

Ethical Statement: The authors are accountable for all aspects of the work in ensuring that questions related to the accuracy or integrity of any part of the work are appropriately investigated and resolved. All participants were recruited and all samples were collected with appropriate informed consent from all participants or their guardians. The study was approved by the ethics committee of the Second Xiangya Hospital of Central South University (No. 2019-Research-40) and was conducted in accordance with the Declaration of Helsinki (as revised in 2013).

Open Access Statement: This is an Open Access article distributed in accordance with the Creative Commons Attribution-NonCommercial-NoDerivs 4.0 International License (CC BY-NC-ND 4.0), which permits the noncommercial replication and distribution of the article with the strict proviso that no changes or edits are made and the original work is properly cited (including links to both the formal publication through the relevant DOI and the license). See: https://creativecommons.org/licenses/by-nc-nd/4.0/.

\section{References}

1. Ramli R, Reddy M, Oliver N. Artificial Pancreas: Current Progress and Future Outlook in the Treatment of Type 1 Diabetes. Drugs 2019;79:1089-101.

2. Shao F, Zheng P, Yu D, et al. Follicular helper T cells in type 1 diabetes. FASEB J 2020;34:30-40.

3. Weng J, Zhou Z, Guo L, et al. Incidence of type 1 diabetes in China, 2010-13: population based study. BMJ 2018;360:j5295.

4. Mayer-Davis EJ, Lawrence JM, Dabelea D, et al. Incidence Trends of Type 1 and Type 2 Diabetes among Youths, 2002-2012. N Engl J Med 2017;376:1419-29. 
5. Kir S, Beddow SA, Samuel VT, et al. FGF19 as a postprandial, insulin-independent activator of hepatic protein and glycogen synthesis. Science 2011;331:1621-4.

6. Cariello M, Piglionica M, Gadaleta RM, et al. The Enterokine Fibroblast Growth Factor 15/19 in Bile Acid Metabolism. Handb Exp Pharmacol 2019;256:73-93.

7. Perry RJ, Lee S, Ma L, et al. FGF1 and FGF19 reverse diabetes by suppression of the hypothalamic-pituitaryadrenal axis. Nat Commun 2015;6:6980.

8. Ryan KK, Kohli R, Gutierrez-Aguilar R, et al. Fibroblast growth factor-19 action in the brain reduces food intake and body weight and improves glucose tolerance in male rats. Endocrinology 2013;154:9-15.

9. Tang MJ, Su JB, Xu TL, et al. Serum fibroblast growth factor 19 and endogenous islet beta cell function in type 2 diabetic patients. Diabetol Metab Syndr 2019;11:79.

10. Kuzuya T. Early diagnosis, early treatment and the new diagnostic criteria of diabetes mellitus. Br J Nutr 2000;84 Suppl 2:S177-81.

11. Zhu M, Xu K, Chen Y, et al. Identification of Novel T1D Risk Loci and Their Association With Age and Islet Function at Diagnosis in Autoantibody-Positive T1D Individuals: Based on a Two-Stage Genome-Wide Association Study. Diabetes Care 2019;42:1414-21.

12. Xiao Y, Xu A, Law LS, et al. Distinct changes in serum fibroblast growth factor 21 levels in different subtypes of diabetes. J Clin Endocrinol Metab 2012;97:E54-8.

13. Executive Summary of The Third Report of The National Cholesterol Education Program (NCEP) Expert Panel on Detection, Evaluation, And Treatment of High Blood Cholesterol In Adults (Adult Treatment Panel III). JAMA 2001;285:2486-97.

14. Xiao Y, Xu A, Hui X, et al. Circulating lipocalin-2 and retinol-binding protein 4 are associated with intima-media thickness and subclinical atherosclerosis in patients with type 2 diabetes. PLoS One 2013;8:e66607.

15. Xiao Y, Liu L, Xu A, et al. Serum fibroblast growth factor 21 levels are related to subclinical atherosclerosis in patients with type 2 diabetes. Cardiovasc Diabetol 2015;14:72.

16. Xiao Y, Xiao X, Xu A, et al. Circulating adipocyte fatty acid-binding protein levels predict the development of subclinical atherosclerosis in type 2 diabetes. J Diabetes Complications 2018;32:1100-4.

17. Schmid A, Leszczak S, Ober I, et al. Short-term and divergent regulation of FGF-19 and FGF-21 during oral lipid tolerance test but not oral glucose tolerance test. Exp Clin Endocrinol Diabetes 2015;123:88-94.
18. Koelfat KV, Bloemen JG, Jansen PL, et al. The portaldrained viscera release fibroblast growth factor 19 in humans. Physiol Rep 2016;4:e13037.

19. Hickey GL, Grant SW, Dunning J, et al. Statistical primer: sample size and power calculations-why, when and how? Eur J Cardiothorac Surg 2018;54:4-9.

20. Tang R, Zhong T, Wu C, et al. The Remission Phase in Type 1 Diabetes: Role of Hyperglycemia Rectification in Immune Modulation. Front Endocrinol (Lausanne) 2019;10:824.

21. Li X, Cao C, Tang X, et al. Prevalence of Metabolic Syndrome and Its Determinants in Newly-Diagnosed Adult-Onset Diabetes in China: A Multi-Center, Cross-Sectional Survey. Front Endocrinol (Lausanne) 2019;10:661.

22. Somm E, Jornayvaz FR. Fibroblast Growth Factor 15/19: From Basic Functions to Therapeutic Perspectives. Endocr Rev 2018;39:960-89.

23. Degirolamo C, Sabba C, Moschetta A. Therapeutic potential of the endocrine fibroblast growth factors FGF19, FGF21 and FGF23. Nat Rev Drug Discov 2016;15:51-69.

24. Fon Tacer K, Bookout AL, Ding X, et al. Research resource: Comprehensive expression atlas of the fibroblast growth factor system in adult mouse. Mol Endocrinol 2010;24:2050-64.

25. Potthoff MJ, Boney-Montoya J, Choi M, et al. FGF15/19 regulates hepatic glucose metabolism by inhibiting the CREB-PGC-1alpha pathway. Cell Metab 2011;13:729-38.

26. Marcelin G, Jo YH, Li X, et al. Central action of FGF19 reduces hypothalamic AGRP/NPY neuron activity and improves glucose metabolism. Mol Metab 2013;3:19-28.

27. Hwang JH, Perseghin G, Rothman DL, et al. Impaired net hepatic glycogen synthesis in insulin-dependent diabetic subjects during mixed meal ingestion. A 13C nuclear magnetic resonance spectroscopy study. J Clin Invest 1995;95:783-7.

28. Fujikawa R, Ito C, Kira S, et al. Longitudinal examination of pancreatic $\beta$-cell function in Japanese individuals. $J$ Diabetes Investig 2020;11:70-4.

29. Li X, Wu D, Tian Y. Fibroblast growth factor 19 protects the heart from oxidative stress-induced diabetic cardiomyopathy via activation of AMPK/Nrf2/HO-1 pathway. Biochem Biophys Res Commun 2018;502:62-8.

30. Siljander H, Honkanen J, Knip M. Microbiome and type 1 diabetes. EBioMedicine 2019;46:512-21.

31. Knip M, Siljander H. The role of the intestinal microbiota in type 1 diabetes mellitus. Nat Rev Endocrinol 
2016;12:154-67.

32. Zheng X, Huang F, Zhao A, et al. Bile acid is a significant host factor shaping the gut microbiome of diet-induced obese mice. BMC Biol 2017;15:120.

33. Fiorucci S, Distrutti E. Bile Acid-Activated Receptors, Intestinal Microbiota, and the Treatment of Metabolic Disorders. Trends Mol Med 2015;21:702-14.

34. Han H, Li Y, Fang J, et al. Gut Microbiota and Type 1 Diabetes. Int J Mol Sci 2018;19:995.

35. Fang Q, Li H, Song Q, et al. Serum fibroblast growth factor 19 levels are decreased in Chinese subjects with impaired fasting glucose and inversely associated with fasting plasma glucose levels. Diabetes Care 2013;36:2810-4.

36. Hu X, Xiong Q, Xu Y, et al. Association of serum fibroblast growth factor 19 levels with visceral fat accumulation is independent of glucose tolerance status. Nutr Metab Cardiovasc Dis 2018;28:119-25.

37. Zhang J, Li H, Bai N, et al. Decrease of FGF19 contributes to the increase of fasting glucose in human in an insulin-independent manner. J Endocrinol Invest 2019;42:1019-27.

38. Stejskal D, Karpisek M, Hanulova Z, et al. Fibroblast growth factor-19: development, analytical characterization and clinical evaluation of a new ELISA test. Scand J Clin Lab Invest 2008;68:501-7.

39. Lai Y, Wang H, Xia X, et al. Serum fibroblast growth factor 19 is decreased in patients with overt hypothyroidism and subclinical hypothyroidism. Medicine (Baltimore) 2016;95:e5001.

40. Zhou M, Learned RM, Rossi SJ, et al. Therapeutic FGF19 promotes HDL biogenesis and transhepatic cholesterol efflux to prevent atherosclerosis. J Lipid Res 2019;60:550-65.

Cite this article as: $\mathrm{Hu} \mathrm{J}$, Tang Y, Liu H, Li Y, Li X, Huang G, Xiao Y, Zhou Z. Decreased serum fibroblast growth factor 19 level is a risk factor for type 1 diabetes. Ann Transl Med 2021;9(5):376. doi: 10.21037/atm-20-5203
41. Babaknejad N, Nayeri H, Hemmati R, et al. An Overview of FGF19 and FGF21: The Therapeutic Role in the Treatment of the Metabolic Disorders and Obesity. Horm Metab Res 2018;50:441-52.

42. Schaap FG, Trauner M, Jansen PL. Bile acid receptors as targets for drug development. Nat Rev Gastroenterol Hepatol 2014;11:55-67.

43. Sonne DP, van Nierop FS, Kulik W, et al. Postprandial Plasma Concentrations of Individual Bile Acids and FGF19 in Patients With Type 2 Diabetes. J Clin Endocrinol Metab 2016;101:3002-9.

44. Byun S, Jung H, Chen J, et al. Phosphorylation of hepatic farnesoid X receptor by FGF19 signaling-activated Src maintains cholesterol levels and protects from atherosclerosis. J Biol Chem 2019;294:8732-44.

45. Hao Y, Zhou J, Zhou M, et al. Serum levels of fibroblast growth factor 19 are inversely associated with coronary artery disease in chinese individuals. PLoS One 2013;8:e72345.

46. Gallego-Escuredo JM, Gomez-Ambrosi J, Catalan V, et al. Opposite alterations in FGF21 and FGF19 levels and disturbed expression of the receptor machinery for endocrine FGFs in obese patients. Int J Obes (Lond) 2015;39:121-9.

47. Friedrich D, Marschall HU, Lammert F. Response of fibroblast growth factor 19 and bile acid synthesis after a body weight-adjusted oral fat tolerance test in overweight and obese NAFLD patients: a non-randomized controlled pilot trial. BMC Gastroenterol 2018;18:76.

48. Lundasen T, Galman C, Angelin B, et al. Circulating intestinal fibroblast growth factor 19 has a pronounced diurnal variation and modulates hepatic bile acid synthesis in man. J Intern Med 2006;260:530-6. 